\title{
El síndrome de Ofelia como asociación única: encefalitis límbica neoplásica y linfoma de Hodgkin
}

\author{
Julio César López-Valdés, ${ }^{1}$ Eduardo González-Negrete ${ }^{2}$ y Arturo Llanes-Ojeda ${ }^{1}$ \\ 'Universidad Autónoma de Tamaulipas. Facultad de Medicina de Tampico "Dr. Alberto Romo Caballero", Tampico, Tamaulipas; ${ }^{2}$ Universidad \\ Nacional Autónoma de México, Unidad de Posgrado, Ciudad de México, México
}

Apreciable editor:

Agradecemos plenamente el interés hacia el artículo "Implicaciones psiquiátricas y neurológicas en la literatura shakespeariana. Breve análisis", comentado en una carta a Gaceta Médica de México. Asimismo, nos es grato extender un amplio reconocimiento por la información que se aporta en la misiva, la cual los autores del artículo reconocen haber desconocido hasta antes de la misma.

Cabe mencionar que si bien el síndrome de Ofelia puede no ser una contribución propia del conocimiento inherente a la era del Bardo, es un ejemplo del alcance alegórico de su figura literaria como prototipo para evocar las características propias de una enfermedad o entidad clínica carente de nombre; igualmente, ejemplifica como un sinfín de médicos recurren a la literatura para hacer referencia a sus descubrimientos u observaciones.

Tras haber leído un poco más sobre el "síndrome de Ofelia", nos complace ahondar en ciertos detalles pertinentes a dicha entidad. Aunque el nombre otorgado por Carr $^{2}$ a la descripción del caso de su hija alude a una entidad clínica asociada con el linfoma de Hodgkin, es necesaria la desambiguación entre dicho síndrome y la encefalitis límbica neoplásica, la cual corresponde a un síndrome neurológico paraneoplásico sugerido por Russell ${ }^{3}$ en 1961; en $70 \%$ de las ocasiones el síndrome se asocia con carcinoma de células pequeñas, aunque también puede estar relacionado con tumores testiculares, cáncer uterino y de mama. ${ }^{4}$ Por tal motivo, quizás es necesario limitar el uso del epónimo shakesperiano solo a la asociación definida del síndrome paraneoplásico mencionado y la presencia de linfoma de Hodgkin, tal cual hizo Carr, ${ }^{2}$ quien en el texto original, a manera de anécdota describe cómo recordó a la damisela danesa tras la solicitud de la valoración psiquiátrica para su hija Jane por parte de su amigo y médico familiar.

Ahora, es necesario mencionar que si bien Carr $^{2}$ sugirió la presencia de moléculas similares a neurotransmisores producidas por tumores como la causa de los fenómenos neurológicos, fue Russell, en su texto "Encephalomyelitis and carcinomatous neuropathy", ${ }^{3}$ quien por primera vez propuso la asociación directa de los síntomas neurológicos con un proceso inmunológico mediado por anticuerpos no descritos para la época.

Dejando de lado lo anterior, consideramos pertinente realizar la siguiente aclaración: también existe el "complejo de Ofelia", término acuñado por el filósofo francés Gastón Bachelard (1884-1962) para el mal d'amour, en el que Ofelia funge como una figura metafórica de la materialización de las emociones mediante el suicidio ("ahogarse en emociones"). ${ }^{5}$

\section{Bibliografía}

1. López-Valdés JC, Miranda-Hernández A, Medina-Medina JA. Implicaciones psiquiátricas y neurológicas en la literatura shakespeariana. Breve análisis. Gac Med Mex. 2018;154(5):613-616.

2. Carr I. The Ophelia syndrome: memory loss in Hodgkin's disease. Lancet. 1982;1(8276):844-845.

3. Russell DS. Encephalomyelitis and carcinomatous neuropathy. En: Van Bogaert L, Radermecker J, Lowenthal A, editores. The encephalitides. Ámsterdam: Elsevier; 1961; p. 131-135.

4. Gil-Ortega M, Hernández-Pardo E, Gil-Ortega I, Gil-Ortega D, Quesada-Córcoles J, Gutiérrez-Lara G, et al. Encefalitis límbica paraneoplásica y cáncer de pulmón. An Med Interna (Madrid), 2004;21(3):35-36.

5. Browning DA. The Weeping Brook: The Ophelia complex in Lorca's poetry. Romance Notes; 2013;53(1):73-82.
Gac Med Mex. 2019;155:109-109

Disponible en PubMed www.gacetamedicademexico.com 